\title{
ADVANCING THE FRONTIERS OF X-RAY CRYSTALLOGRAPHY IN ENGINEERED SINGLE CRYSTALS: ENANTIOMORPHIC STRUCTURES
}

\author{
Morelhão, S. L. ${ }^{1}$,; Amirkanyan, Z. G. ${ }^{1}$; Remédios, C. M. R. ${ }^{2}$ \\ ${ }^{1}$ Instituto de Física, Universidade de São Paulo, São Paulo, SP, Brazil \\ ${ }^{1}$ Faculdade de Física, Universidade Federal do Pará, Belém, PA, Brazil \\ *morelhao@if.usp.br
}

Crystallography has its roots in diffraction methods of X-rays, neutrons, and electrons, where resolution in determining crystal structures depends on refinement procedures. Collection of large data sets of diffracted intensities and adjustment of parameters in model structures to simulate the experimental intensities are the very basic steps common to all these refinement procedures. Accuracy in determining structural features is therefore dictated by reliability or goodness-of-fitting values. Besides intensity measurements, which are measurements of amplitude of structure factors, physical measurements of structure factor phases are also possible, but for X-rays only [1]. In this work, we demonstrate how to use theoretical phase values for planning an experiment to inspect features susceptible to changes when designing single crystals, such as in doping of optical crystals. Atomic disordering at one particular group of atoms, small change in atomic fractional coordinates due to internal stresses, and enantiomorphism are some of these features. Emphasis is on how accurate we need to describe the structure for succeeding in this demonstration, establishing precedents for a discussion on realistic perspectives of using physical phase measurements as a new procedure in crystallography of more complex crystals.

[1] Z.A. Amirkhanyan, C.M.R. Remédios, Y.P. Mascarenhas, S.L. Morelhão. J. Appl. Cryst. 47, 160 (2014). doi:10.1107/S1600576713028677 